\title{
Democracia y economía: determinantes políticos del desempeño económico en América Latina $^{1}$
}

\author{
Soraia Marcelino Vieira \\ Universidade Federal Fluminense (UFF) \\ Pedro Cavalcante \\ Instituto de Pesquisa Econômica Aplicada (Ipea)
}

Augusto Neftali Corte de Oliveira

Fundação de Economia e Estatística (FEE)

En las últimas décadas, América Latina ha atravesado profundas transformaciones políticas y económicas. A pesar de los análisis restringidos a la influencia de periodos electorales, prevalece un vacío acerca del impacto de la dinámica política democrática sobre el desempeño de las naciones, fundamentalmente sobre los efectos de la ideología partidaria y de la competición electoral. Con base en los datos de 18 países latinoamericanos, entre 1990 y 2010, los modelos estadísticos refuerzan la importancia de la política en la explicación de los resultados económicos, caracterizados por las tasas de crecimiento del PIB, inflación y desempleo, aunque en menor medida de lo que la teoría preconiza.

Palavras clave: desarrollo económico, democracia, poder político, política económica

\section{Democracia e economia: determinantes políticos do desempenho econômico na América Latina}

Nas últimas décadas, América Latina vem atravessando profundas transformações políticas e econômicas. Apesar das análises restritas à influencia de períodos eleitorais, prevalece um vazio acerca do impacto da dinâmica política democrática sobre o desempenho das nações, fundamentalmente sobre os efeitos da ideologia partidária e da competição política. Com base em dados de 18 países latino-americanos entre 1990 e 2010, os modelos estatísticos reforçam a importância da política para a explicação dos resultados econômicos, caracterizados pelas taxas de crescimento do PIB, inflação e desemprego, ainda que em menor medida do que preconiza a teoria.

Palavras-chave: desenvolvimento econômico, democracia, poder político, política econômica

\footnotetext{
${ }^{1}$ Tradución al Español de Juan Vicente Bachiller Cabria
} 


\section{Democracy and economy: determinants of economic performance in Latin America}

In the last decades, Latin America has experienced intense political and economic changes. Since the bulk of studies have been focused on the influence of election periods (political business cycle), a lack of research prevails concerning the effects of the democratic politics, such as party ideology and electoral competition, on the nation's performances. Based on panel data of 18 Latin American countries from 1990 to 2010, regression results reinforce the importance of politics explaining economic performance, characterized by the GDP growth, inflation and unemployment rates, although the political effects are lesser extent than the theory advocates.

Keywords: economic development, democracy, political power, economic policy 


\section{Introducción}

El principal objetivo de este artículo es analizar de forma comparada los determinantes, sobre todo de naturaleza política, del desempeño macroeconómico de las naciones latinoamericanas en el reciente periodo predominantemente democrático.

En las dos últimas décadas América Latina viene atravesando profundas transformaciones institucionales pautadas, principalmente, por la apertura de las economías nacionales y por la democratización. Aunque prevalezca la creencia de la población en la democracia como la mejor forma de gobierno, a los latinoamericanos les continúan preocupando diversas cuestiones estructurales. Las demandas por un mayor desarrollo económico y mejores servicios sociales, así como la necesidad de controlar la inflación continúan en la agenda. En la dimensión política, destaca el restablecimiento de los derechos políticos y, consecuentemente, la vuelta de procesos electorales relativamente estables y periódicos.

A pesar del notorio avance en la comprensión de las causas y del desarrollo de la democracia en la región, poco se sabe acerca de los impactos efectivos de la dinámica democrática sobre el desempeño de las naciones.

La literatura está restringida a análisis respecto de la influencia de periodos electorales sobre el comportamiento de los políticos, enfocada en la teoría de los ciclos políticos de negocios (political business cycle). Con ello, prevalece una laguna acerca de los efectos de la ideología partidaria y de la competición electoral, variables centrales en la medida en que muchos países de la región vienen experimentando alternancia de partidos en el poder, inclusive con la ascensión al poder de partidos de izquierda, como también aumento de la competición en comicios nacionales. Así, la presente investigación aplica regresión múltiple con datos longitudinales de dieciocho países latinoamericanos entre 1990 y 2010 para investigar los determinantes de sus resultados económicos, caracterizados por las tasas de crecimiento del PIB, inflación y desempleo.

Además de esta introducción, el artículo está organizado en otras tres secciones. Primero, se realizará una breve discusión acerca de los cambios políticos y económicos que la región ha atravesado recientemente. Seguidamente, el estudio desarrolla un análisis exploratorio de las variables de desempeño económico, contextualiza y presenta el modelo de análisis, como también debate los principales resultados. Por último, son discutidas algunas conclusiones. 


\section{Mudanzas políticas y económicas en América Latina}

El final del siglo 20 fue marcado por una serie de mudanzas políticas y económicas en todo el mundo. En América Latina no fue diferente. La región experimentó la apertura democrática en varios países, y, al mismo tiempo, enfrentó graves problemas económicos relacionados con la inflación, la deuda externa y las sucesivas crisis ${ }^{2}$ que perjudicaron el crecimiento y dejaron un impacto social negativo en la mayoría de los países de la región. La tercera ola de democratizaciones, por tanto, llegó a América Latina deslegitimando los gobiernos autoritarios y restituyendo la democracia en un ambiente de gran inestabilidad económica y social. ¿Cómo promover el cambio institucional, consolidar la democracia y lidiar con las restricciones económicas?

Mientras los gobernantes que Ilegaron al poder en América Latina enfrentaron estos desafíos, a nivel mundial se consolidaba la opción por las políticas neoliberales. En los países desarrollados, la crisis económica de precios de la década de 1970 llevó a la interpretación de que los regímenes de bienestar poseían efectos perjudiciales sobre la inversión en el proceso productivo (NOTERMANS, 2000). La incapacidad de la socialdemocracia en presentar una solución para esta crisis, así como la ascensión de los gobiernos de Marageth Thatcher en Inglaterra y Ronald Reagan en Estados Unidos, Ilevaron a las políticas del neoliberalismo a ocupar una posición incontrastable en el pensamiento de gobiernos y operadores económicos mundiales. El peso del Estado y su capacidad de intervención en el mercado fueron problemas colocados en la pauta de las reformas.

Las condiciones macroeconómicas regionales y el contexto internacional impusieron a los países en desarrollo una difícil elección: adoptar los preceptos macroeconómicos capitaneados por las agencias multilaterales -Banco Mundial y Fondo Monetario Internacional-, o buscar una salida alternativa y enfrentar el riesgo de perder acceso a los organismos de crédito. Lo que se observó fue que, bajo las presiones de los condicionantes externos, muchos países de la región optaron por la adopción del recetario ortodoxo. En las palabras de Cruz (2007, p. 27) "Ios países latinoamericanos vienen enfrentando, desde el inicio de los años ochenta, una coyuntura severamente adversa desde el punto de vista de su autonomía nacional".

Junto con el contexto internacional, es posible observar que los gobiernos que llegaron democráticamente al poder en este periodo fueron formados por

\footnotetext{
2 Las crisis que afectaron a los países en desarrollo a mediados de la década de 1990 - México en 1995; los Tigres Asiáticos en 1997, Rusia en 1998, Argentina en 2001- causaron un fuerte impacto sobre las economías latinoamericanas.
} 
coaliciones de derecha o centro-derecha, que aceptaron las orientaciones de los mecanismos internacionales y adoptaron la agenda ortodoxa. Como revela Stokes (2001), incluso algunos presidentes que fueron electos con promesas centradas en la seguridad económica acabaron adoptando políticas neoliberales en sus gobiernos. Así, la década de 1990 presenta una inclinación hacia la derecha en la política económica de la región. De acuerdo con Diniz (2007), América Latina se tornó un laboratorio de aplicación de los preceptos neoliberales. Stiglitz (2002) argumenta que América Latina "se volvió el mejor alumno del Consenso de Washington, con Argentina y Chile apareciendo como los pupilos más aplicados" (STIGLITZ, 2002, p. 245). Aunque en estos dos países las reformas neoliberales se iniciaran durante los regímenes militares, los gobiernos electos que siguieron no promovieron mudanzas de curso significativas.

Para América Latina, la adopción de reformas liberales radicales fue prescrita no sólo como un remedio contra la inflación, sino también para la solución de los problemas de crecimiento económico (RODRIK, 2006). Así, los gobiernos que optaron por adoptar la agenda neoliberal buscaron, por medio del equilibrio macroeconómico, apertura externa, mercados libres y privatización, un modelo capaz de garantizar estabilidad y prosperidad (VALDÉs, 2008, p. 208). Aunque las medidas neoliberales hayan permitido una recuperación en los índices de crecimiento en la década de 1990, su grado fue inferior al del periodo 1950-1980.

En el campo social, la adopción de medidas ortodoxas tuvo fuertes consecuencias sobre las clases populares. Los porcentuales de empleo formal se redujeron, al mismo tiempo que creció el número de desempleados y de personas viviendo en la pobreza. Con sistemas de políticas sociales deficitarias -en que grandes contingentes de población permanecieron, a lo largo de la historia, excluidos ya bien de la provisión de protección social, ya bien de relaciones de trabajo estableslas reformas que disminuyeron la capacidad del Estado en actuar en el proceso económico se tornaron todavía más socialmente onerosas en América Latina. Los efectos políticos del crecimiento económico lento y de los costos sociales de las medidas neoliberales llegaron al inicio del siglo 21 reflejándose en el desencanto de la población latinoamericana. Conforme Valdés:

A principios del siglo XXI, se observa en toda la región un serio problema de frustración de expectativas. Existe la percepción de que el crecimiento económico de América Latina después de las reformas fue reducido. Por distintas causas, observando la situación que prevaleció en el siglo XXI, ese conjunto de reformas no produjo una mejora substantiva y permanente en un segmento importante de la población de muchos países latinoamericanos (VALDÉs, 2008, p. 208). 
Después del fracaso de las políticas neoliberales es posible observar un viraje en el péndulo de la disputa política, esta vez en dirección a la izquierda. Los partidos de izquierda que se estructuraron con las aperturas democráticas se presentaron como una opción para los electores descontentos con la plataforma económica ortodoxa y los costos sociales acarreados (LORA; OLIVEIRA, 2005; BAKer; Greene, 2011). De acuerdo con Pannizza (2006), una verdadera marea roja alcanzó a América Latina. Partidos de izquierda y centro izquierda llegaron al poder en Argentina, Bolivia, Brasil, Chile, Costa Rica, Ecuador, Nicaragua, Panamá, Perú, Venezuela y Uruguay.

Aunque tengan sus especificidades marcadas por la trayectoria de los partidos, liderazgos y por el contexto específico de cada país, algunos elementos comunes pueden ser observados en los gobiernos progresistas que llegaron al poder al final de la década de 1990 e inicio de los años 2000. Uno de ellos es la oposición al neoliberalismo, identificada tanto con mudanzas en la política económica, como con preocupaciones más directas con el bien estar de la población, la inclusión social y la disminución de la desigualdad. En palabras de Silva (2010), "el antineoliberalismo sirvió simbólicamente de "farol" y de denominador común aglutinador, en medio de la "neblina" de las grandes transformaciones ocurridas en la última trayectoria histórica" (SILVA, 2010, p. 5). En este contexto, el Estado volvió a ser visto como un ente regulador necesario para la manutención de la estabilidad económica en América Latina. Una importante medida fue la alteración de los precios relativos en el mercado internacional, la cual provocó un efecto positivo, lo que favoreció las balanzas comerciales y promovió una nueva ruta en dirección al crecimiento, aunque resultando más tímida que la observada en el periodo anterior. No obstante, las tasas de crecimiento económico no se presentan de manera uniforme en la región.

\section{Determinantes del desempeño económico en América Latina}

\section{Desempeño en el contexto democrático: análisis descriptivo}

América Latina viene experimentando a lo largo del tiempo oscilaciones en su desempeño macroeconómico. Después de un periodo de considerable crecimiento durante las décadas de 1960 y 1970 , lo que se observa es un periodo de inestabilidad y alto grado de variabilidad entre las experiencias nacionales. Considerando estos factores, ¿cómo mensurar el desempeño económico en la región? La literatura acerca del tema es muy diversa, aunque, para los fines de esta investigación optamos por tres importantes indicadores macroeconómicos que, en gran medida, reflejan los resultados de las políticas económicas de los gobiernos nacionales. Se 
trata de crecimiento del producto interior bruto (PIB), tasa de inflación y variación en el índice de desempleo.

Además de ser buenos indicadores de los resultados alcanzados por la política económica del gobierno, estas variables también ejercen impacto en el cotidiano de la sociedad, lo que las torna todavía más interesantes en los análisis de los determinantes del comportamiento y consecuentemente del desempeño de los gobernantes.

La variable crecimiento es medida mediante el porcentual de crecimiento anual del producto interior bruto de los 18 países estudiados, con datos proporcionados de la United States Agency for International Development (2011). La media del indicador observado en el período de veinte años para el conjunto de los países de la investigación fue de $3,8 \%$. Todos los países presentan un saldo positivo, siendo que el menor fue obtenido por México $(2,8 \%)$ y el mayor por Panamá $(5,6 \%)$. No existen mayores diferencias si se consideran los bloques económicos o comerciales de la región: los países del Mercosur alcanzaron un crecimiento del 3,3\%, los países de la Comunidad Andina (inclusive Venezuela y Chile), 3,8\%, y los países abarcados por el Tratado de Libre Comercio de América Central y República Dominicana (CAFTA-RD) obtuvieron un crecimiento de 3,9\%.

De hecho, como puede observarse a partir de la figura más abajo, la obtención de crecimiento económico predominó durante el periodo de la investigación (1990-2010). Considerando los 18 países, en los veinte años aquí analizados, en apenas 45 casos (13\%) hubo retracción económica. Aunque la mayor parte haya pasado por al menos un año con caída en el producto interior bruto, tres países pasaron todo el periodo de crecimiento constante: Bolivia, Panamá y Guatemala. Por otro lado, algunos países experimentaron retracción en número mayor de años: Venezuela en siete años, Argentina en seis, Uruguay en cinco. Destacan dos casos con más de dos años de recesión, ocurrida en Argentina y en Uruguay, ambos entre los años de 1999 y 2002. Reflejo de las crisis internacionales, los peores años para la economía latinoamericana fueron los de 2009 y 1999, con nueve y ocho países en recesión, respectivamente. 
Figura 1 - Crecimiento económico en América Latina, 1990-2010

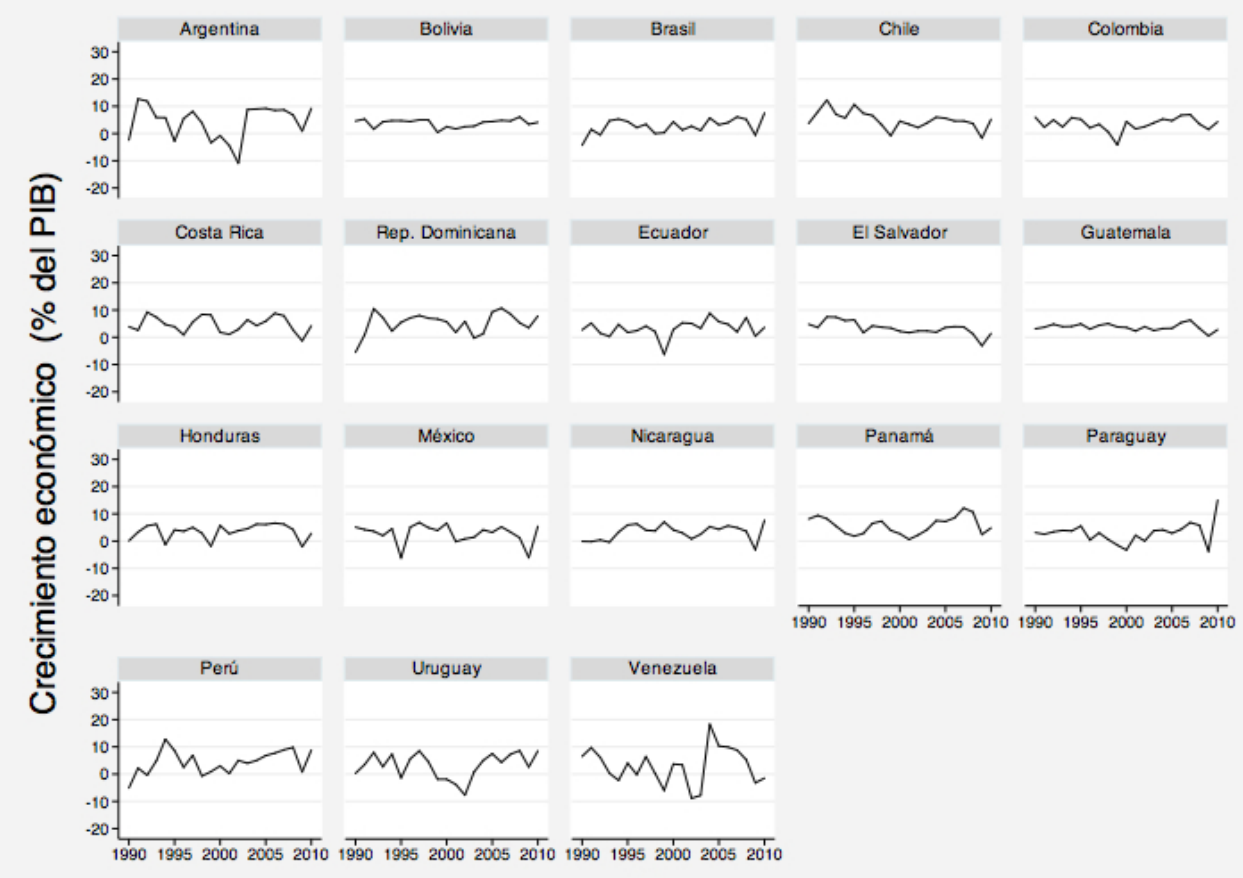

Año

Fuente: United States Agency for International Development (2011). Elaboración propia.

En cuanto a la inflación, la variable seleccionada refleja los índices oficiales de los gobiernos para la media anual de inflación medida por índice de precios al consumidor para los 18 países de la investigación, conforme datos del Banco Interamericano de Desarrollo (2012). En este conjunto, cuatro países alcanzaron, en al menos un año, índices de inflación superiores al 200\% después de 1990 Argentina, Brasil, Nicaragua y Perú3 ${ }^{3}$. Por otro lado, 13 países enfrentaron al menos tres años consecutivos de inflación superior al 15\% entre 1990 y 2010. En la mayoría de los casos, los problemas inflacionarios fueron controlados en el inicio de la década de 1990, como es posible visualizar en la Figura 2 a continuación. Seis países no registraron tres años consecutivos de inflación superior al 15\% después de 1995, Brasil no lo registró después de 1996, Honduras y Uruguay después de 1997, Colombia después de 1998, México después de 1999 y Ecuador después de 2001. Argentina se destaca por presentar índices negativos entre 1999 y 2001 ,

\footnotetext{
${ }^{3}$ Para no comprometer las estimativas de los modelos, diez casos extremos -tasas de inflación encima de 200\%fueron sustituidos por la media. Como se trata de menos del $3 \%$ del total de observaciones, se estima que tal opción no disminuye la validad interna del análisis.
} 
aunque el único caso disonante en lo que concierne al control de la inflación es el de Venezuela -a lo largo de los años 2000, el país continúa a ostentar índices de inflación superiores al 20\%.

Figura 2 - Tasa de inflación en América Latina, 1990-2010

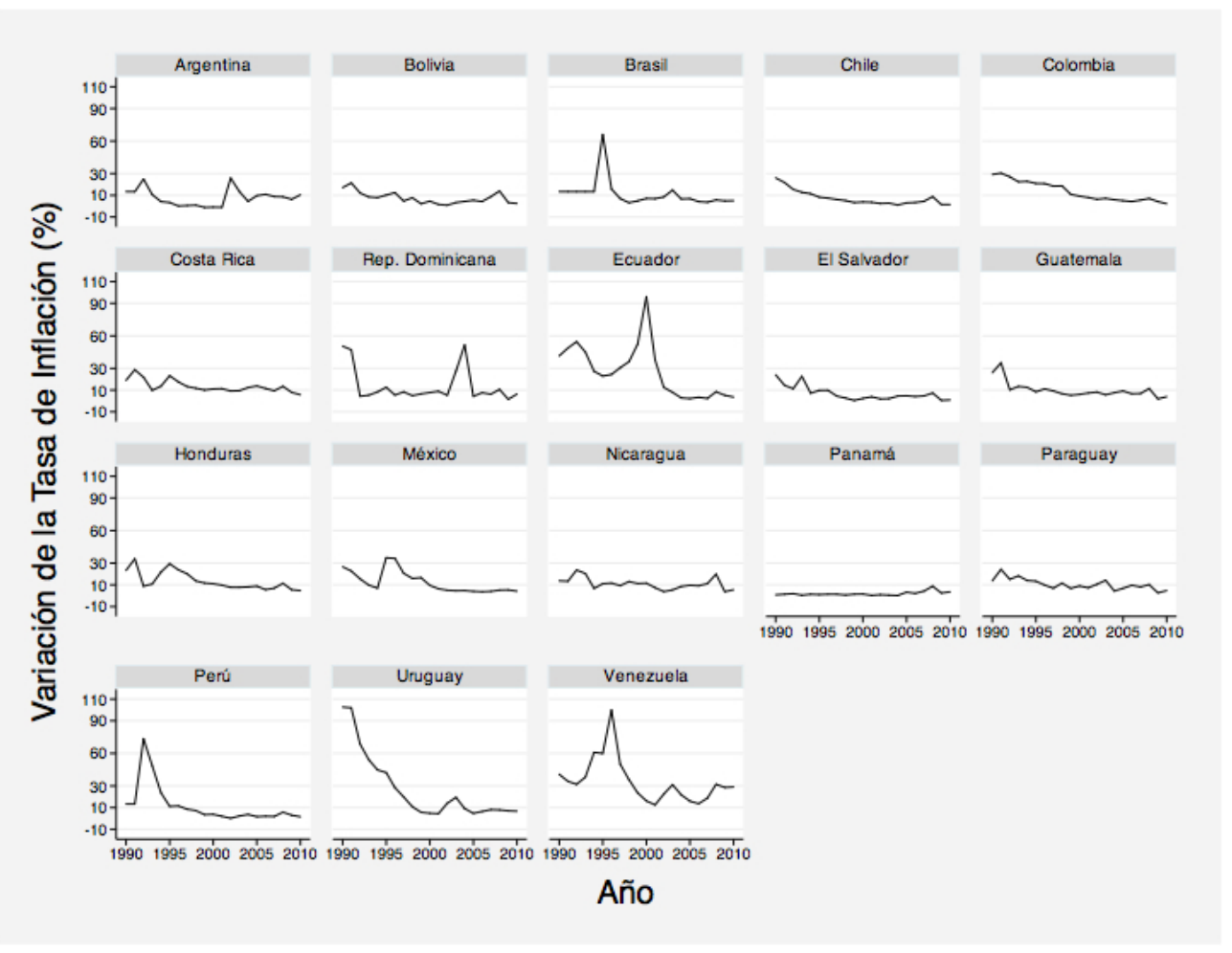

Fuente: Banco Interamericano de Desarrollo (2012). Elaboración propia.

En lo que concierne al desempleo, fueron utilizados datos del Banco Interamericano de Desarrollo (2012), suplementados, para los casos ausentes, con datos del United States Agency for International Development (2011). La investigación opta por utilizar la variación de la tasa anual de desempleo en relación al año anterior. Se estima que esa es una medida más precisa que la simple tasa de desempleo, dado que posibilita mensurar los resultados de las políticas económicas entre los años, como también excluye la cuestión del desempleo estructural. Como puede observarse a partir de la figura de abajo, los países presentan una variación de la tasa anual de desempleo constante en el periodo de la investigación (1990-2010). La situación más grave de aumento del desempleo ocurrió en Argentina (6\%) en 1995. Por otro lado, los mejores resultados en cuanto a la caída de desempleo pueden ser observados en Ecuador en 2000 (-5,4\%), en República Dominicana en 1994 (-3,9\%) y en Uruguay en 2004 (-3,8\%). 
Figura 3 - Variación del índice de desempleo en América Latina, 1990-2010

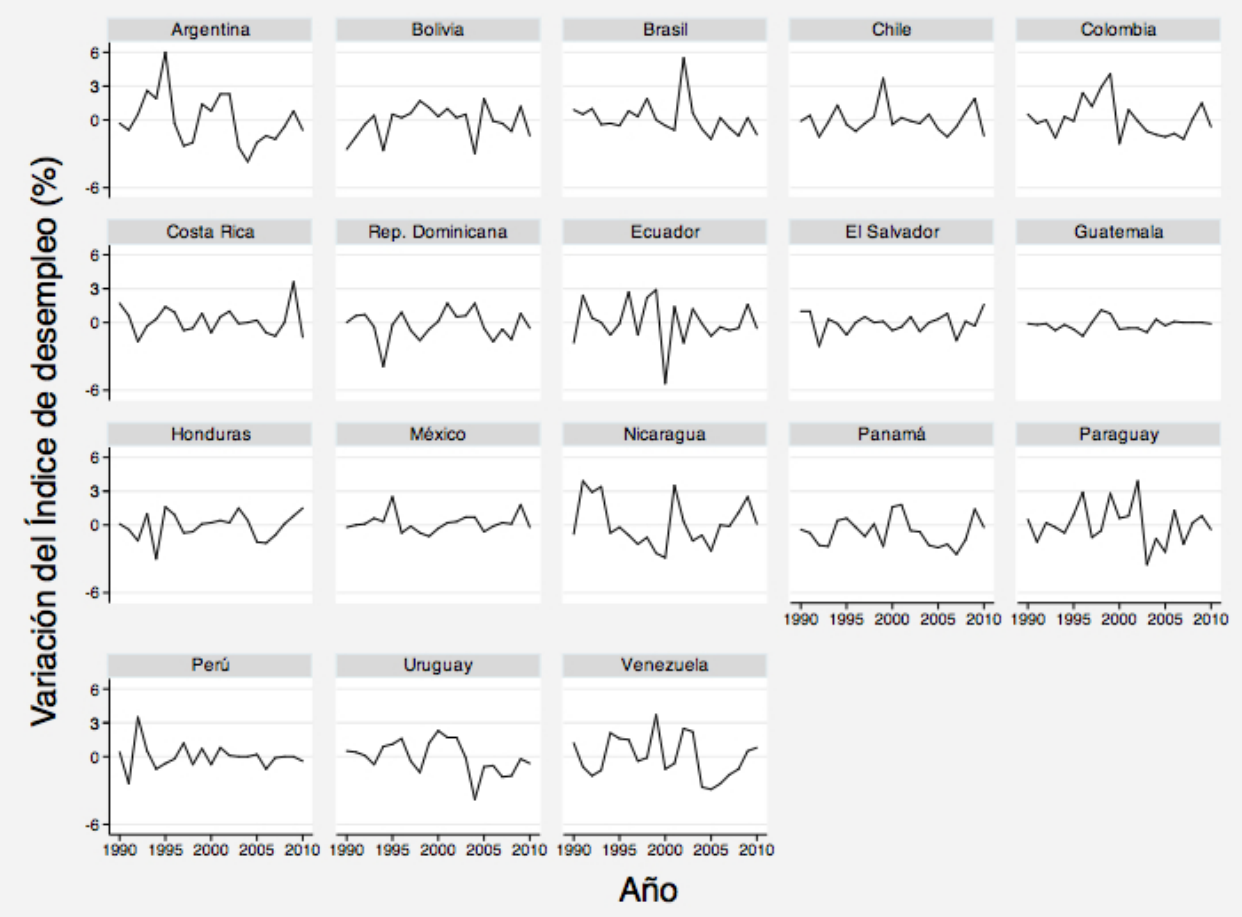

Fuente: Banco Interamericano de Desarrollo (2012); United States Agency for International Development (2011). Elaboración propia.

\section{Modelo explicativo}

El objetivo principal de este trabajo es evaluar el impacto de la dinámica política, en especial competición electoral e ideología, sobre el desempeño económico de los gobiernos nacionales en América Latina durante el presente periodo democrático. Este tipo de abordaje no es nuevo en las Ciencias Sociales. Desde los años 1970, una serie de trabajos viene investigando comparativamente los efectos de factores de naturaleza política sobre los resultados de la política económica, especialmente en los países de la Organización para la Cooperación y el Desarrollo Económico (OCDE) (CASTLES, 1978; CASTLES; McKinlay, 1979; CAMERon, 1978). Esta línea de investigación tiene como premisa la importancia del papel de la intencionalidad de las decisiones sobre los resultados de la economía, como se evidencia en la afirmación de Douglas Hibbs (1977, p. 1487): “los resultados macroeconómicos no son totalmente endógenos, aunque, obviamente, son influenciados en gran medida por elecciones políticas de corto y largo plazo". 
En las últimas décadas todavía prevalece la ausencia de consenso acerca de la efectiva influencia política sobre la economía. En este contexto, Imbeau, Pétryy Lamari (2001), a partir de una extensa investigación bibliográfica, clasifican la literatura en dos vertientes: "escuela de la convergencia", y los adeptos de la perspectiva de que "la política importa". Los primeros argumentan que las sociedades industrializadas del siglo 20 se tornaron cada vez más similares, encarando los mismos tipos de problemas y aplicando los mismos tipos de soluciones. Por tanto, las diferencias políticas, culturales e institucionales poco sirven para explicar las variaciones de las políticas públicas. En contrapartida, la segunda vertiente, aunque no niegue la influencia de factores socioeconómicos, preconiza la existencia de correlación entre variables relativas a la dinámica política y a los resultados de políticas públicas. Ese debate continúa latente en los abordajes comparados entre naciones que, en los últimos años, analizan una gran variedad de temas más allá del crecimiento económico (BJORNSKOV, 2005), incluyendo también el proceso de desregulación del mercado (PORTAFKE, 2010), liberalización de la industria (BELLOC; NICITA, 2011) y privatización (BJORNSKOV; PORTRAFKE, 2011).

En el sentido de aplicar ese debate al caso latinoamericano, el presente artículo busca testar dos hipótesis relacionadas con el funcionamiento del sistema democrático. El argumento central de la teoría de la democracia electoral es que las elecciones y sus aspectos subyacentes influencian el comportamiento de los políticos, y, por consiguiente, el desempeño de los gobiernos. En la perspectiva de la accountability 4 , la democracia tiende a producir gobiernos controlados y responsivos a la población, cuyos partidos políticos actúan como actores centrales dentro del proceso electoral libre, justo y frecuente.

Autores como Castles (1982), Klingemann, Hofferbert y Budge (1994) y Powell (2009) apuntan hacia el papel fundamental de los partidos políticos en el proceso democrático, en la medida en que se organizan y presentan en las elecciones paquetes de políticas ideológicamente coherentes, permitiendo así que la democracia conecte la preferencia de los ciudadanos y la política pública. Para otros autores, sin embargo, esta capacidad de los partidos se debilitó debido a la ampliación de los espacios aislados de la política en los gobiernos y por la falta de claridad en la distinción ideológica entre las agremiaciones, entre otros factores (MAIR, 2007). Aunque los partidos no desempeñan un papel controlador de la política pública por medio de la ideología, la competición electoral periódica puede incentivar la accountability en la medida en que permite al electorado juzgar el desempeño de los gobernantes, generando recompensas y puniciones que estimulan al gobierno a respetar los intereses de los ciudadanos (MANIN; PRZEWORSKI; STOKES, 1999).

\footnotetext{
${ }^{4}$ Accoutability entendida como la obligación de transparencia y prestación de cuentas de los representantes a sus electores.
} 
Así, la presente investigación estudia esos dos importantes factores políticos relacionados con la accountability: competición electoral e ideología partidaria. La variable competición electoral es empleada para testar si el grado de disputa en las elecciones posee impacto sobre el desempeño de los gobiernos locales y, por tanto, promueve accountability. En otras palabras, esperamos que cuanto más disputada la elección para la presidencia en los países latinoamericanos, mejor el desempeño de los gobiernos en la economía -mayor crecimiento, menor inflación y menor desempleo, o sea en un ambiente de alta competitividad, donde hay más incertidumbre en cuanto a la posibilidad de permanecía en el poder, buscando hacer un incumbent o reelegirse, el gobierno hace un mayor esfuerzo para garantir el mejor desempeño económico y social posible

Un segundo aspecto central en la literatura es el impacto de la ideología del partido del presidente sobre el desempeño gubernamental. Esta investigación se basa en el posicionamiento de Downs (1999), que ordena la izquierda y la derecha de acuerdo con la visión sobre la intervención estatal en la economía. En las democracias industriales, por lo menos hasta la década de 1970, los gobiernos de izquierda estuvieron asociados a una política económica direccionada para el crecimiento económico y la generación de empleo, en detrimento del control de la inflación (ESPING-ANDERSEN, 1998; HIBBS, 1977). En la misma dirección, Przeworski $(1985$,$) defiende que "la ideología que orienta a la derecha es el antiestatismo [...]$ basado en la creencia de la racionalidad del mercado [...] mientras que la izquierda posterior a la segunda guerra mundial preconiza el programa keynesiano de pleno empleo y equidad" (PRZEWORSKI, 1985, p. 205).

Según este raciocinio, el criterio básico de la diferenciación remite al papel del Estado y del mercado, mediante el cual los partidos de izquierda presentarían una postura más intervencionista a favor del empleo y del crecimiento económico. Para ello, son utilizadas las estimativas de posicionamiento ideológico de investigación con los parlamentarios de cada país investigado (cámaras bajas), en las cuales los mismos sitúan a los partidos entre las posiciones cero (más a la izquierda) y diez (más a la derecha). Las expectativas en términos de resultados de las economías para la posición del partido del presidente en esta variable son las siguientes: cuanto más bajo (izquierda), más crecimiento económico y menos desempleo; cuanto más alto (derecha), menor la inflación.

Empleamos también otras dos variables políticas: "año electoral" e "índice de derechos políticos" de Freedom House. La primera se fundamenta en la teoría del ciclo político de negocios (political business cycle) y pretende medir los efectos de la agenda del ciclo electoral sobre el desempeño de la economía. Es decir, si ocurre tendencia de mayor crecimiento e inflación y reducción de desempleo en periodos 
de elecciones para mejorar la imagen de gestión frente a los electores (NORDHAUS, 1975; TABELLINI; PERSSON, 1999). En cuanto a los derechos políticos, su aplicación en el modelo tiene como objetivo evaluar si el régimen o el nivel de democratización del país ejerce o no efectos sobre los resultados alcanzados en términos de política económica, algo rechazado por algunos académicos (PRZEWORSKI et al., 2000).

Otros factores también pueden contribuir a explicar el desempeño de las naciones, por eso utilizamos un conjunto de factores de orden económico como control. El "porcentual de las exportaciones en relación al PIB" y el "grado de inversión externa" pueden ser indicativos de abertura económica y de liberalización de la economía $y$, por consiguiente, favorecen un mejor desempeño de este sector. De la misma forma, las "parcelas de gastos sociales" y de "la industria sobre el PIB" son utilizadas para testar si la mayor propensión de inversiones en ese área y el mayor dinamismo económico pueden reflejarse en mejores resultados económicos. Finalmente, para capturar la coyuntura internacional, el "crecimiento de la economía de los países de la OCDE" es empleado en el modelo, dada la histórica dependencia de las naciones latinoamericanas en relación con los países que componen este bloque.

\section{Resultados}

En el sentido de estimar los efectos de los determinantes políticos sobre el desempeño económico en América Latina, fueron aplicadas las técnicas tradicionales de regresión econométrica para datos en panel: efectos fijos y efectos aleatorios. Ambostipos de modelos para análisis con datos de panel poseen ventajas y desventajas. De acuerdo con Wooldrige (2006), el estimador de efectos fijos es eficiente cuando los errores idiosincráticos son seriamente no correlacionados y homoscedásticos. Los modelos de efectos aleatorios son más apropiados en situaciones en que el efecto no observado es no correlacionado con todas las variables explicativas. En todos los casos, con todo, los resultados de los testes de Hausman apuntan hacia la consistencia de las estimativas obtenidas a través del método de efectos fijos.

Como es posible percibir en la Tabla 1 a continuación, de modo general, los resultados empíricos indican poca capacidad explicativa de los modelos, lo que es reiterado por sus bajos coeficientes de determinación. En el primer modelo, determinantes del crecimiento económico, si por un lado las evidencias confirman la hipótesis de la influencia de la ideología, por otro, la relativa a la competición electoral es rechazada.

Por lo que respecta al impacto de la ideología partidaria, las evidencias empíricas favorecen la interpretación de que la alternancia de poder en la región, más específicamente, las victorias de partidos más izquierdistas generaran mejores desempeños económicos. El coeficiente estimado indica que cuanto menor es la 
medida de ideología empleada (más a la izquierda el gobierno), mayores tienden a ser las variaciones positivas del PIB. Es decir, un punto menos en la medida ideológica del partido de gobierno tiende a generar de media cerca de un 0,6 más de crecimiento del PIB nacional.

En cuanto a la competición electoral, su coeficiente positivo y significante indica justamente el esperado teóricamente, es decir, cuanto más apretadas son las elecciones presidenciales en América Latina, mejores los desempeños en términos de crecimiento del PIB de los candidatos electos en el transcurrir de sus mandatos. Desde un punto de vista sustantivo, sin embargo, los efectos son de hecho residuales en la medida en que la variación de un desvío padrón encima de la media tiende a reflejarse en menos de 0,001 en la variación del crecimiento económico.

En relación con las demás variables políticas, año electoral no demuestra ser un factor influyente en esta dimensión, así como tampoco el indicador de derechos políticos empleado en el modelo. De entre las variables de control, solamente el nivel de industrialización de la nación parece afectar a las tasas de crecimiento económico en América Latina durante el periodo analizado. Ese efecto, no obstante, es relevante en la medida en que el incremento de un uno por ciento en la participación de la industria en el PIB refleja un 0,3\% de más de crecimiento en la economía.

El modelo de los determinantes de la inflación también indica significancia estadística de la variable de competición electoral. En este caso, el coeficiente es significativo y negativo, lo cual converge con el efecto esperado. En otras palabras, cuanto más disputada es la elección mejor tiende a ser el desempeño del presidente en el combate a la inflación, más específicamente un punto porcentual en el margen de victoria menos genera una reducción en el índice inflacionario de 0,15, manteniendo los demás factores constantes.

Por otro lado, la segunda hipótesis relativa al impacto de la ideología no se confirma en el análisis de la inflación, así como el hecho de realizarse elecciones en el año o de prevalecer un régimen político más democrático. Además, el nivel de gasto en el área social se mostró significativo y con un efecto expresivo, en vista de que el incremento de un desvío padrón en relación con la media de los gastos sociales repercute en aproximadamente un $2 \%$ de más en los índices de inflación anual, lo que en gran medida es comprensible.

Finalmente, en el modelo de los determinantes del desempeño, así como en los demás, la variable de competición electoral presenta efectos estadísticamente significativos. En este caso, mientras tanto, aunque con efectos más residuales que en la inflación, las evidencias muestran la relación negativa del coeficiente estimado.

Así, puede afirmarse que cuanto mayor es la competición en las elecciones en América Latina, mejores tienden a ser los resultados en la política de empleo. En 
los demás factores de carácter político no se observa impacto significativo de la ideología, año electoral y derechos políticos. En cuanto a los controles, el aumento de participación de la exportación en el PIB parece repercutir en menores índices de desempleo, en términos sustantivos, un aumento de un punto porcentual en la participación de las exportaciones sobre el PIB tiende a generar una reducción de 0,06 en las tasas de desempleo de las naciones, de media.

Tabla 1 - Determinantes del desempeño económico en América Latina, 1990-2010

\begin{tabular}{|c|c|c|c|}
\hline & $\begin{array}{l}\text { Crecimiento } \\
\text { Económico }\end{array}$ & Inflación & Desempleo \\
\hline \multirow{2}{*}{$\begin{array}{l}\text { Competición } \\
\text { Electoral }\end{array}$} & $0,04 *$ & $-0,15^{* *}$ & $-0,01 * *$ \\
\hline & $(0,02)$ & $(0,07)$ & $(0,01)$ \\
\hline \multirow{2}{*}{ Ideologia } & $-0,56^{*}$ & 1,67 & 0,01 \\
\hline & $(0,32)$ & $(1,04)$ & $(0,12)$ \\
\hline \multirow{2}{*}{ Año Electoral } & $-0,15$ & $-1,63$ & 0,02 \\
\hline & $(0,55)$ & $(1,78)$ & $(0,21)$ \\
\hline \multirow{2}{*}{$\begin{array}{l}\text { Índice de Derechos } \\
\text { Políticos }\end{array}$} & $-0,02$ & 1,56 & $-0,01$ \\
\hline & $(0,33)$ & $(1,09)$ & $(0,12)$ \\
\hline \multirow{2}{*}{ Exportación } & 0,00 & 0,11 & $-0,06 * * *$ \\
\hline & $(0,06)$ & $(0,20)$ & $(0,22)$ \\
\hline \multirow{2}{*}{ Inversión Externa } & 0,18 & $-0,44$ & $-0,05$ \\
\hline & $(0,11)$ & $(0,19)$ & $(0,44)$ \\
\hline \multirow{2}{*}{ Gastos Sociales } & 0,15 & $-3,77 * * *$ & 0,03 \\
\hline & 0,14 & $(0,47)$ & $(0,55)$ \\
\hline \multirow{2}{*}{ Industrialización } & $0,30 * * *$ & $-0,20$ & $-0,05$ \\
\hline & $(0,08)$ & $(0,46)$ & $(0,03)$ \\
\hline \multirow{2}{*}{ Crecimiento OCDE } & 0,18 & $-1,21$ & $-0,04$ \\
\hline & $(0,26)$ & 0,084 & $(0,09)$ \\
\hline \multirow{2}{*}{ Constante } & $-5,67$ & 56,63 & $2,71 *$ \\
\hline & $(4,04)$ & $(13,07)$ & $(1,54)$ \\
\hline $\mathbf{R}^{2}$ & 0,01 & 0,005 & 0,01 \\
\hline $\mathbf{N}$ & 269 & 269 & 269 \\
\hline Teste de Hausman & $24,13 * *$ & $83,90 * * *$ & $20,57^{* *}$ \\
\hline
\end{tabular}

Banco Interamericano de Desarrollo (2012), Center for Latin American Studies (2012), Center of Democratic Performance (2012), Comisión Económica para América Latina (2012), Freedom House (2011), Observatório de Elites Parlamentarias en América Latina (2012), United States Agency for International Development (2011). Elaboración propia.

Notas: Los coeficientes estimados y errores estándar aparecen entre paréntesis. Significación estadística: *significa valor de $\mathrm{p}<0,1, * *$ P-valor $<0,05, * * *$-valor $<0,01$. 


\section{Consideraciones finales}

El artículo se propone desarrollar una investigación sobre los efectos de la dinámica política en las últimas dos décadas sobre el desempeño macroeconómico de las naciones latinoamericanas. La temática se torna todavía más relevante en la medida en que este periodo fue marcado por un conjunto de transformaciones políticas e institucionales profundas que culminaron en inéditas reconfiguraciones de las fuerzas políticas en la región. En esta dirección, la investigación procuró testar si el grado de competición en las elecciones presidenciales y la postura ideológica de los partidos gobernantes ejercieron efectos sobre el desempeño de las economías en América Latina, retratadas por el crecimiento del PIB, la inflación y el desempleo.

Los modelos estadísticos, en síntesis, refuerzan la importancia de estos factores en la explicación de los resultados económicos, aunque en menor medida de lo que la teoría democrática preconiza. Por lo que respecta a la competición electoral, la hipótesis de que cuanto más disputada son las elecciones para la presidencia de los países latinoamericanos, mejor el desempeño de los gobiernos es confirmada para la inflación y el desempleo. Por su parte, la hipótesis del papel más proactivo de los nuevos partidos de izquierda puede ser constatado en el análisis del crecimiento económico. En relación con las otras dos variables de carácter político, primero podemos afirmar que en el periodo analizado el grado de democratización del país, mensurado por el índice de derechos políticos, no importa, del mismo modo que no se observó impacto de comportamiento oportunista en años electorales en los resultados de la economía de la región, lo que converge con la literatura acerca del tema (BORSANI, 2003).

Otro aspecto interesante de los modelos está relacionado con la influencia de factores estructurales sobre el desempeño económico de las naciones. Se percibe que el impacto de las variables seleccionadas no solamente es poco frecuente, sino que, principalmente, éstas varían de acuerdo con cada tipo de resultado macroeconómico observado.

En suma, podemos concluir que es innegable la relevancia de inclinarse hacia la dimensión política en la explicación del desempeño económico de los países. Mientras tanto, reconocemos que esta tarea no es trivial, sobre todo en función de la complejidad de la temática. En ese sentido, creemos que una mayor profundización en los análisis requiere tanto la aplicación de nuevos métodos y modelos estadísticos, como también la opción por metodología cualitativa que propicie investigaciones más detalladas acerca del impacto de las diversas configuraciones políticas e institucionales sobre los resultados económicos de las naciones latinoamericanas. Desde un punto de vista substantivo, cabe resaltar que los estudios comparados sobre 
los determinantes del desarrollo en esta región se presentan como un terreno fértil de investigación, principalmente si ampliamos el foco incluyendo otras dimensiones como la social y la de la sustentabilidad, por ejemplo.

\section{Referencias bibliográficas}

BAKER, A; GreENE, K. F. The Latin American left's mandate: freemarket policies and issue voting in new democracies. World Politics, v. 63, n. 01, p. 43-77, 2011, Janeiro.

Banco INTERAMERICANo de DeSARRollo. Latin Macro Watch Database. 2012. Disponible en: http://www.iadb.org/Research/LatinMacroWatch/. Acceso en: 6 de mar. de 2012.

BELLOC, F; NICITA, A. Liberalization-privatization paths: policies and politics, Departamental Working Papers 32, Department of Economics, Business and Statistics at Università degli Studi di Milano, 2011.

BJornskov, C. Does political ideology affect economic growth? Public Choice, v.123, n. 12, p. 133-146, 2005.

BJoRnskov, C; Potrafke, N. Politics and privatization in Central and Eastern Europe: a panel data analysis. In: Economics of Transition, v. 19, n. 2, p. 201-230, 2011.

BORSANI, H. Eleições e economia: instituições políticas e resultados macroeconômicos na América Latina (1979-1998). Belo Horizonte, Rio de Janeiro: UFMG, IUPERJ, 2003. CAMERon, D. The expansion of the Public Economy: a comparative analysis. The American Political Science Review, v. 72, n. 4, p. 1243-1261, 1978.

CASTLES, F. (Org). The impact of parties: politics and policies. London: Sage, 1982.

CASTLES, F. The social democratic image of society. London: Routledge; Kegan Paul, 1978.

CASTLES, F.; MCKINLAY, R. Does politics matter? An analysis of public welfare commitment in advanced democratic states. European Journal of Political Research, v.7, n. 2, p. 169-186, 1979.

Center for latin American Studies. Political Database of the Americas. 2012. Disponible en: http://pdba.georgetown.edu/Elecdata/elecdata.html. Acceso en: 6 de mar. de 2012.

Center of Democratic Performance. Election Results Archive. 2012. Disponible en: http://www.binghamton.edu/cdp/era/countries/. Acceso en: 6 de mar. de 2012.

COMISIÓN ECONÓMICA PARA AMÉRICA LATINA. Bases de Datos y Publicaciones Estadísticas. 2012. Disponible en: http://estadisticas.cepal.org/. Acceso en: 6 de mar. de 2012.

CRUZ, S. Trajetórias: capitalismo neoliberal e reformas econômicas nos países da periferia. São Paulo: Editora Unesp, 2007.

DıNıZ, E. Globalização Estado e Desenvolvimento. Dilemas do Brasil no novo milênio. Rio de Janeiro: FGV, 2007.

Downs, A. Uma Teoria Econômica da Democracia. São Paulo: Edup. 1999. 
ESPING-ANDERSEN, G. The three worlds of Welfare Capitalism. Princeton: Princeton University, 1998.

FREEDOM HOUSE. Freedom in the world. 2011. Disponible en: http://www. freedomhouse.org. Acceso en: 6 de mar. de 2012.

HIBBS, D. Political parties and macroeconomic policy. The American Political Science Review, v. 71, n. 4, p. 1467-1487, 1977.

IMBEAU, L; PÉTRY, F; LAMARI, M. Left-right parties ideology and government policies: a meta-analysis. European Journal of Political Research, v. 40, p. 1-29, 2001.

Klingemann, H; Hofferbert, R; Budge, I. Parties, policies, and democracy. Boulder, San Francisco, Oxford: Westview, 1994.

LORA, E; OLIVEIRA, M. The electoral consequences of the Washington Consensus, Economia, v. 5, n. 2, primavera, p. 1-61, 2005.

MAIR, P. The challenge to party government. EUI Working Papers. San Domenico di Fiesole, 2007.

Manin, B; PrzeWorski, A; Stokes, S. Introduction. In: Przeworski, A; Stokes, S; MANIN, B (Eds.). Democracy, accountability, and representation. Cambridge/Nova York: Cambridge University Press, 1999.

Nordhaus, W. The political business cycle. Review of Economic Studies, n. 42, p. 169-190, 1975.

Notermans, T. Money, markets, and state: social democratic economic policies since 1918. Cambridge: Cambridge University, 2000.

Observatorio de Elites Parlamentarias en América latina. Series Temporales. 2012. Disponible en: http://americo.usal.es/oir/. Acceso en: 6 de mar. de 2012.

PAnizza, F. La Marea Rosa. Análise de Conjuntura - Observatório Político Sulamericano, n. 8, agosto, 2006.

POTRAFKE, N. Does government ideology influence deregulation of product markets? Empirical evidence from OECD countries. Public Choice, v. 143, n. 1, p.135-155, 2010.

POWELL Jr., G. Aggregating and representing political preferences. In: BOIX, C; STOKES, S. (org.). Comparative Politics. Oxford: Oxford University, 2009. p. 653-677.

PRZEWORSKI, A. Capitalism and social democracy. Cambridge: Cambridge University Press, 1985.

PrZeworski, A; Alvarez, M. E; Cheibub, J. A.; LIMongI, F. Democracy and development: political institutions and well-being in the world, 1950-1990. Cambridge: Cambridge University Press, 2000.

RoDRIK, D. Goodbye Washington Consensus, Hello Washington Confusion? Journal of Economic Literature, v. 44, n. 4, p. 973-987, 2006.

SILVA, F. Até onde vai a "onda rosa? Análise de Conjuntura - Observatório Político Sul-americano, n.o 2, fev. 2010.

StIGLITZ, J. A globalização e seus malefícios: a promessa não cumprida de benefícios globais. São Paulo: Editora Futura, 2002.

STOKES, S. C. Mandates and democracy: neoliberalism by surprise in Latin America. 
Cambridge: Cambridge University, 2001.

TABELLINI, G; PERSSON, T. The size and scope of government: comparative politics with rational politicians. European Economic Review, v. 43, p. 699-735, 1999.

United States Agency for International Development. Latin America and the Caribbean Selected Economic and Social Data. 2011. Disponible en: http://lac.eads. usaidallnet.gov/. Acceso en: 6 mar. 2012.

VALDÉs, J. G. Pinochet's economists: the Chicago school of economics in Chile. Cambridge, UK: Cambridge University Press, 2008.

WOOLRIDGE, J. Introdução à econometria: uma abordagem moderna. São Paulo: Editora Thomson, 2006.

\section{Soraia Marcelino Vieira}

Doutora em Ciência Política pelo Instituto de Estudos Sociais e Políticos da Universidade do estado do Rio de Janeiro (Uerj). Atualmente é professora Adjunta na Universidade Federal Fluminense (UFF) e membro titular da Facultad Latinoamericana de Ciencias Sociales España- Flacso, Espanha. Contato: soraiamv@id.uff.br

\section{Pedro Cavalcante}

Doutor em Ciência Política (UnB) com pós doutorado da School of International and Public Affairs (SIPA) da Columbia University e Estágio de Doutorando (University of California - San Diego - UCSD). Atualmente atua como pesquisador do Instituto de Pesquisa Econômica Aplicada. Professor do Mestrado Profissional em Administração Pública do Ppga/UnB, Idp e Ipea. Contato: cavalcante.pedro@gmail.com

\section{Augusto Neftali Corte de Oliveira}

Doutor em Ciência Política pela Universidade Federal do Rio Grande do Sul (UFRGS). Atualmente é Pesquisador em Ciência Política da Fundação de Economia e Estatística Siegfried Emanuel Heuser (FEE/RS). Contato: ancolive@gmail.com 
RSP 